DOI: 10.17516/1997-1397-2020-13-2-197-212

УДК 519.21

\title{
Rotationally-axisymmetric Motion of a Binary Mixture with a Flat Free Boundary at Small Marangoni Numbers
}

\author{
Victor K. Andreev* \\ Institute of Computational Modelling SB RAS \\ Krasnoyarsk, Russian Federation \\ Siberian Federal University \\ Krasnoyarsk, Russian Federation \\ Natalya L. Sobachkina ${ }^{\dagger}$ \\ Siberian Federal University \\ Krasnoyarsk, Russian Federation
}

Received 06.092019, received in revised form 06.11. 2019, accepted 06.02.2020

\begin{abstract}
Rotationally-axisymmetric motion of a binary mixture with a flat free boundary at small Marangoni numbers is investigated. The problem is reduced to the inverse linear initial-boundary value problem for parabolic equations. Using Laplace transformation properties the exact analytical solution is obtained. It is shown that a stationary solution is the limiting one with the growth of time if there is a certain relationship between the temperature of the solid wall and the external temperature of the gas. If there is no connection, the convergence to the stationary solution is broken. Some examples of numerical reconstruction of the temperature, concentration and velocity fields are given, which confirm the theoretical conclusions.
\end{abstract}

Keywords: binary mixture, free boundary, inverse problem, the pressure gradient, the stationary solution, Laplace transformation, thermal Marangoni number.

Citation: V.K.Andreev, N.L.Sobachkina, Rotationally-axisymmetric Motion of a Binary Mixture with a Flat Free Boundary at Small Marangoni Numbers, J. Sib. Fed. Univ. Math. Phys., 2020, 13(2), 197-212. DOI: 10.17516/1997-1397-2020-13-2-197-212.

\section{Introduction}

The main purpose of this work is to construct an exact solution of the inverse initial boundary value problem of rotationally symmetric motion of a viscous heat-conducting binary mixture with a flat free boundary at small Marangoni numbers, as well as a numerical solution of the problem. The movement is caused by a non-stationary pressure gradient.

It is well known that for small Marangoni numbers, the momentum equation can be simplified by discarding convective acceleration. Such movements are called crawling. Similar simplifications can be obtained for the energy and concentration transfer equations. One of these problems, considered in paper [1], is devoted to the study of solving the thermodiffusion equations of a special type that describes the two-dimensional motion of a binary mixture in a flat channel. In the resulting initial boundary value problem, the analog of the Marangoni number is the Reynolds number. Assuming that this number is small, the problem becomes linear. Its solution is found using trigonometric Fourier series that converge rapidly for any given time.

There are a lot of theoretical works concerning convective movements in flat layers with a free boundary. R. V. Birikh's exact stationary solutions to the problem of thermocapillary convection

\footnotetext{
*andr@icm.krasn.ru

†sobachkinanat@mail.ru

(c) Siberian Federal University. All rights reserved
} 
in a flat horizontal layer are well known in work [2]. One solution describes the flow in the band $-h<x<0$, both borders of which are solid walls, and in the second - the upper border of the band is free, subject to the action of thermocapillary forces. The solutions were widely used and cited [3-15]. In a number of these works [6,7,10,13-15], the flat Benard-Marangoni convection of a viscous incompressible liquid was studied in the Oberbeck-Bussinesque model. A characteristic feature of the obtained solutions is the one-dimensional velocity coordinates, and the temperature and pressure fields are three-dimensional. In the work [13], an exact solution was obtained near the point of the temperature extremum at zero Grasgoff number. The found solution serves as an initial approximation for constructing solutions for Grasshoff numbers greater than zero. In works $[14,15]$ of the initial boundary value problem describing non-stationary layered flows of the Benard-Marangoni convection in an infinitely extended flat layer, the existence of counterflows in the liquid layer was found. The presence of counterflows is equivalent to the presence of stagnant points, which indicates the existence of a local extremum of the kinetic energy of the liquid.

In this paper, in the absence of external forces, we study the creeping axisymmetric motion of a mixture with a flat free boundary with a Hiemenz type velocity field [16]. Here the inverse problem arises, since the non-stationary pressure gradient is also the desired function.

\section{Statement of the problem}

We consider the axisymmetric motion of an infinite horizontal plane layer of a viscous heatconducting binary mixture bounded by a solid wall $z=0$ and a free boundary $z=l(t)$ (see Fig. 1). Let $\mathbf{u}(\mathbf{x}, t)$ is the velocity vector, $p(\mathbf{x}, t)$ is the pressure, $\theta(\mathbf{x}, t), c(\mathbf{x}, t)$ are deviations from the average values temperature and concentration values of the mixture under conditions of complete weightlessness. The process is described by a system of equations of thermodiffusion motion [17]:

$$
\begin{aligned}
& \frac{d \mathbf{u}}{d t}+\frac{1}{\rho} \nabla p=\nu \Delta \mathbf{u}, \quad \operatorname{div} \mathbf{u}=0, \\
& \frac{d \theta}{d t}=\chi \Delta \theta, \quad \frac{d c}{d t}=d \Delta c+\alpha d \Delta \theta,
\end{aligned}
$$

where $\rho$ is the average density, $\nu$ is the kinematic viscosity, $\chi$ is the thermal diffusivity, $d$ is the diffusion coefficient, $\alpha$ is the thermodiffusion coefficient (Soret coefficient); $d / d t=\partial / \partial t+\mathbf{u} \cdot \nabla$ is the full time derivative, $\Delta$ is the Laplace operator.

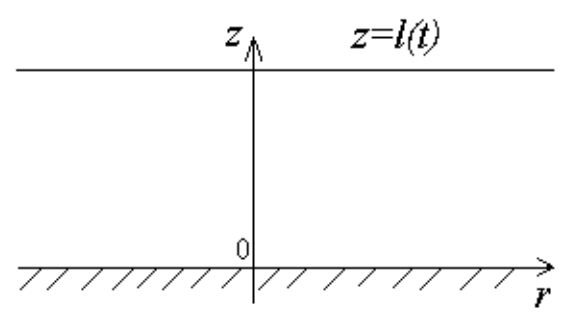

Fig. 1. Diagram of the flow region

Remark 1. The equation of energy from the system (1) does not take into account the term describing the dissipation of kinetic energy. This is due to the fact that the ratio of this term and $\mathbf{u} \cdot \nabla \theta$ for most processes does not exceed $10^{-7}$. In addition, all model parameters are assumed to be constant, and they are reliably determined experimentally.

Let $u(r, z, t), w(r, z, t)$ are projections of the velocity vector in cylindrical coordinate system. 
The solution of the problem is searched for in a special form:

$$
\begin{gathered}
u=r u_{1}(z, t), \quad w=w(z, t), \quad p=p(r, z, t), \quad \theta=a(z, t) r^{2}+b(z, t), \\
c=h(z, t) r^{2}+g(z, t) .
\end{gathered}
$$

A solution of the form (2) is called a Hiemenz type solution [16], in which the velocity field is linear relative to one from the coordinates. It is partially invariant with respect to the five-parameter subgroup generated by the operators $\partial / \partial r, t \partial / \partial r+\partial / \partial u, \partial / \partial \theta, \partial / \partial c, \partial / \partial p[18]$.

Substituting the form (2) into the system of thermodiffusion equations leads to the system (reassign $u_{1} \leftrightarrow u$ ):

$$
\begin{gathered}
r u_{t}+r u^{2}+r w u_{z}+\frac{1}{\rho} p_{r}=r \nu u_{z z} ; \\
w_{t}+w w_{z}+\frac{1}{\rho} p_{z}=\nu w_{z z} \\
2 u+w_{z}=0 \\
a_{t}+2 a u+w a_{z}=\chi a_{z z} ; \\
b_{t}+w b_{z}=\chi\left(4 a+b_{z z}\right) ; \\
h_{t}+2 h u+w h_{z}=d h_{z z}+\alpha d a_{z z} ; \\
g_{t}+w g_{z}=d\left(4 h+g_{z z}\right)+\alpha d\left(4 a+b_{z z}\right),
\end{gathered}
$$

that needs to be solved in the field $t>0,0<z<l(t)$.

It is assumed that the surface tension coefficient $\sigma$ at the free boundary linearly depends on the temperature and concentration

$$
\sigma(\theta, c)=\sigma_{0}-æ_{1}\left(\theta-\theta_{0}\right)-æ_{2}\left(c-c_{0}\right)
$$

where $æ_{1}>0$ is the temperature coefficient of surface tension, $æ_{2}$ is the concentration coefficient of surface tension (usually $æ_{2}<0$, since the surface tension increases with increasing concentration); $\theta_{0}, c_{0}$ are some constant average values.

Boundary conditions on an unknown free boundary $z=l(t)$ for the system $(3)-(9)$ have the form:

$$
\begin{gathered}
\frac{d l}{d t}=w(l(t), t) ; \\
u_{z}=-\frac{2 æ_{1}}{\rho \nu} a-\frac{2 æ_{2}}{\rho \nu} h ; \\
p_{g a s}-p+2 \rho \nu w_{z}=0 ; \\
k a_{z}+\gamma\left(a-a_{g a s}\right)=0 ; \\
k b_{z}+\gamma\left(b-b_{g a s}\right)=0 ; \\
h_{z}+\alpha a_{z}=0 ; \\
g_{z}+\alpha b_{z}=0,
\end{gathered}
$$

where $p_{\text {gas }}, \theta_{\text {gas }}$ are the pressure and the temperature of the surrounding gas; $k, \gamma$ are the thermal conductivity and the heat transfer coefficients. It is assumed that the transfer processes in gas can be neglected. It is assumed that the gas pressure $p_{\text {gas }}$ is constant, and its temperature $\theta_{\text {gas }}$ at the border with the liquid mixture is set by the function of time. Thus, the ratio (10) is the kinematic condition, (11), (12) are tangential and normal dynamic conditions, (13), (14) is a condition for heat exchange with the gas surrounding the mixture, (15), (16) is a condition for the absence of a flow of matter across a free boundary (thus the effect of surfactants on $z=l(t)$ is not taken into account). 
Boundary conditions on a solid wall $z=0$ :

$$
\begin{gathered}
u(0, t)=0, \quad w(0, t)=0, \quad a(0, t)=a(t), \quad b(0, t)=b(t), \\
h_{z}(0, t)+\alpha a_{z}(0, t)=0, \quad g_{z}(0, t)+\alpha b_{z}(0, t)=0 .
\end{gathered}
$$

Initial conditions:

$$
\begin{gathered}
u(z, 0)=u_{0}(z), \quad w(z, 0)=w_{0}(z), \quad a(z, 0)=a_{0}(z), 1 \quad b(z, 0)=b_{0}(z), \\
h(z, 0)=h_{0}(z), \quad g(z, 0)=g_{0}(z), \quad l(0)=l_{0}>0,
\end{gathered}
$$

and the functions $u_{0}, w_{0}, a_{0}, b_{0}$ satisfy the conditions $(17) ; u_{0}$ and $w_{0}$ are connected by equation (5); $u_{0}, a_{0}, h_{0}$ are connected by condition (11); $h_{0}, a_{0}-$ by conditions (15) and (17); $g_{0}, b_{0}$ - by conditions (16) and (17). Thus, the approval conditions are met.

From the equations $(3),(4)$, the pressure gradient $\left(p_{r}, p_{z}\right)$ is expressed:

$$
\begin{gathered}
p_{r}=-r \rho\left(u_{t}+u^{2}+w u_{z}-\nu u_{z z}\right) \\
p_{z}=\rho\left(w_{z z}-w_{t}-w w_{z}\right)
\end{gathered}
$$

The compatibility conditions of the equations (19), (20) are satisfied identically: $p_{r z}=p_{z r}=0$. It follows that the function $u(z, t)$ will be determined from the equation

$$
u_{t}+u^{2}+w u_{z}=\nu u_{z z}+f(t)
$$

and the pressure is restored by the formula

$$
p=-\frac{r^{2}}{2} \rho f(t)+s(z, t)
$$

here $f(t)$ is arbitrary function, and the derivative of the variable $z$ from the function $s(z, t)$ is exactly the right side of the equation (20). The function $s(z, t)$ is considered known if the function $w(z, t)$ is found.

Therefore, the problem is inverse, since the longitudinal pressure gradient $f(t)$ is an unknown function. In the theory of inverse problems, it is called a source function.

\section{Converting to a task in a fixed area}

You can see that the equations (21), (5), (6), (8) are independent of the others. They form a closed initial boundary value problem for defining the functions $u(z, t), a(z, t), h(z, t)$, and $l(t)$. Therefore, we will reduce the task to finding only these functions. To do this, we integrate the equation (5) and exclude the function $w$ in the equations $(21),(6),(8)$. In the resulting system, we introduce dimensionless variables and functions with equalities:

$$
\begin{gathered}
\tau=\frac{\nu t}{l_{0}^{2}}, \quad y=\frac{z}{l(t)}, \quad U=\frac{l_{0}^{2} u}{\nu}, \quad A=\frac{l_{0}^{2} a}{\bar{T}}, \\
A_{\text {gas }}=\frac{l_{0}^{2} a_{g a s}}{\bar{T}}, \quad H(z, t)=\frac{l_{0}^{2} h}{\bar{c}}, \quad L(\tau)=\frac{l(t)}{l_{0}}, \quad F(\tau)=\frac{l_{0}^{4} f(t)}{\nu^{2}},
\end{gathered}
$$

here $\bar{T}, \bar{c}$ are characteristic temperature and concentration.

The result is a task in a fixed area $0<y<1$ :

$$
M(U) \equiv U_{\tau}-(\ln L)_{\tau} y U_{y}-2 U_{y} \int_{0}^{y} U(y, \tau) d y+U^{2}-\frac{1}{L^{2}} U_{y y}-F(\tau)=0
$$




$$
\begin{gathered}
F(U, A) \equiv A_{\tau}-(\ln L)_{\tau} y A_{y}-2 A_{y} \int_{0}^{y} U(y, \tau) d y+2 A U-\frac{1}{\operatorname{Pr} L^{2}} A_{y y}=0 \\
R(U, A, H) \equiv H_{\tau}-(\ln L)_{\tau} y H_{y}-2 H_{y} \int_{0}^{y} U(y, \tau) d y+2 H U- \\
-\frac{1}{\operatorname{Sc} L^{2}} H_{y y}-\frac{\operatorname{Sr}}{\operatorname{Sc} L^{2}} A_{y y}=0 .
\end{gathered}
$$

In (24)-(26), dimensionless parameters are entered: $\mathrm{Sc}=\nu / d$ is Schmidt number, $\mathrm{Sr}=\alpha d \bar{T} / \nu \bar{c}$ is Soret number, $\operatorname{Pr}=\nu / \chi$ is Prandtl number.

The following conditions are met on a solid wall $y=0$ :

$$
U(0, \tau)=0, \quad A(0, \tau)=A(\tau), \quad H_{y}(0, \tau)+\operatorname{Sr} A_{y}(0, \tau)=0 .
$$

On a free boundary $y=1$ :

$$
\begin{gathered}
\frac{d L}{d \tau}=-2 L \int_{0}^{1} U(y, \tau) d y \\
A_{y}+L \operatorname{Bi}\left(A-A_{g a s}\right)=0 ; \\
-\frac{1}{2 L} U_{y}=\operatorname{Ma} A+\operatorname{Mc} H ; \\
H_{y}+\operatorname{Sr} A_{y}=0,
\end{gathered}
$$

where $\mathrm{Bi}=\gamma l_{0} / k$ is the number of Bio; $\mathrm{Ma}=æ_{1} \bar{T} l_{0} / \rho \nu^{2}, \mathrm{Mc}=æ_{2} \bar{c} l_{0} / \rho \nu^{2}$, respectively, the thermal Marangoni number and the concentration Marangoni number.

Initial conditions for $\tau=0$ :

$$
\begin{gathered}
U(y, 0)=U_{0}(y), \quad A(y, 0)=A_{0}(y), \quad H(y, 0)=H_{0}(y), \\
L(0)=1, \quad F(0)=F^{0} \equiv \text { const. }
\end{gathered}
$$

To find an unknown pressure gradient $F(\tau)$ when solving the inverse problem, you need to set an additional condition. As such the condition is an integral redefinition condition, which is written as:

$$
\int_{0}^{1} U d y=0, \quad y=1 .
$$

This is a condition of closed flow. Thus, the flow rate of the liquid mixture through any normal cross-section is zero. Given the conditions (28) and (32), it follows from the integral redefinition condition (33) that the free boundary remains fixed and is equal to $L(\tau)=1$.

\section{Stationary solution}

We will assume that the thermal Marangoni number is Ma $\ll 1$ (the creeping motion), as well as Ma $\sim \mathrm{Mc}$, that is, thermal and concentration effects on a free boundary of the same order. Formally decomposing the functions $U, A, H$ in a row by $\mathrm{Ma}$, we get for the first approximation the problem (24)-(26) with $\mathrm{Ma}=0$. In the equations of momentum, heat transfer, and concentration, the convective terms are discarded. We will consider the steady flow of the liquid. For such a movement, all the required functions do not depend on time; let's denote them by $U^{0}(y), A^{0}(y), H^{0}(y), F^{0}$. Also, on a solid wall, $A(\tau)=A \equiv$ const. Let's write out the corresponding boundary value problem for $0<y<1$, which becomes linear for small Marangoni numbers:

$$
U_{y y}^{0}+F^{0}=0
$$




$$
\begin{gathered}
A_{y y}^{0}=0 ; \\
H_{y y}^{0}+\operatorname{Sr} A_{y y}^{0}=0,
\end{gathered}
$$

with boundary conditions (27)-(31).

When searching for a stationary solution, a fundamental result was obtained. That is, in order for the solutions found to satisfy all boundary conditions, it is necessary and sufficient that the temperature of the solid wall is associated with the external temperature of the gas by a certain condition. The relationship between temperatures is as follows:

$$
A=-\frac{\mathrm{Bi} A_{\text {gas }}^{0}}{\mathrm{Bi}+2}
$$

Then the required functions in the first approximation have the form:

$$
\begin{gathered}
A^{0}(y)=\frac{\operatorname{Bi~} A_{\text {gas }}^{0}(2 y-1)}{\mathrm{Bi}+2} ; \\
H^{0}(y)=\frac{\mathrm{BiSr} A_{\text {gas }}^{0}(1-2 y)}{\mathrm{Bi}+2} ; \\
U^{0}(y)=\frac{\operatorname{Bi} A_{\text {gas }}^{0}(1-\mathrm{MSr})\left(y-1,5 y^{2}\right)}{\mathrm{Bi}+2} ; \\
F^{0}=3 \frac{\mathrm{Bi} A_{\text {gas }}^{0}(1-\mathrm{MSr})}{\mathrm{Bi}+2},
\end{gathered}
$$

where $\mathrm{M}=æ_{2} \bar{c} / æ_{1} \bar{T}$ is a dimensionless parameter equal to the ratio of the thermal Marangoni number to the concentration Marangoni number.

In addition, representations are found for other functions of the General problem, which made a significant contribution to obtaining a certain relationship between temperatures:

$$
\begin{aligned}
& B^{0}(y)=-\frac{2}{3} \alpha_{1} y^{3}-2 \alpha_{2} y^{2}+\beta_{1} y+\beta_{2} ; \\
& G^{0}(y)=-\frac{2}{3} \gamma_{1} y^{3}-2 \gamma_{2} y^{2}+\delta_{1} y+\delta_{2} ;
\end{aligned}
$$

where $\alpha_{1}, \alpha_{2}, \beta_{1}, \beta_{2}, \gamma_{1}, \gamma_{2}, \delta_{1}, \delta_{2}$ are constants defined from boundary conditions (27)-(32):

$$
\begin{gathered}
\alpha_{2}=-\frac{\operatorname{Bi} A_{\text {gas }}^{0}}{\operatorname{Bi}+2}, \quad \alpha_{1}=-2 \alpha_{2}, \quad \beta_{2}=B, \\
\beta_{1}=\frac{\operatorname{Bi}\left(B_{\text {gas }}^{0}-\beta_{2}+\frac{2}{3} \alpha_{1}+2 \alpha_{2}\right)+2 \alpha_{1}+4 \alpha_{2}}{\operatorname{Bi}+1}, \\
\gamma_{1}=-\alpha_{1} \mathrm{Sr}, \quad \gamma_{2}=-\frac{\gamma_{1}}{2}, \quad \delta_{1}=-\beta_{1} \mathrm{Sr}, \quad \delta_{2}=\frac{\alpha_{1} \mathrm{Sr}}{6}+\frac{\beta_{1} \mathrm{Sr}}{2}+C .
\end{gathered}
$$

Here $B$ is the second component of the solid wall temperature for the stationary case, and $C$ is a constant that sets the average cross-section concentration $y=0$.

\section{Determining of the temperature field}

For solution of nonstationary linear problem is used Laplace transform. Believe (assuming the existence of $\left.\tilde{A}, \tilde{A}_{y}, \tilde{A}_{y y}, \tilde{A}_{\text {gas }}[19]\right)$ :

$$
\tilde{A}(y, p)=\int_{0}^{\infty} A(y, \tau) e^{-p \tau} d \tau
$$


then the problem for $A(y, \tau)$ is reduced to the boundary value problem for an ordinary differential equation

$$
\begin{gathered}
\tilde{A}_{y y}-\operatorname{Pr} p \tilde{A}=-\operatorname{Pr} A_{0}(y), \quad 0<y<1 \\
\tilde{A}(0, p)=\tilde{A}(p), \quad y=0 \\
\tilde{A}_{y}+\operatorname{Bi}\left(\tilde{A}-\tilde{A}_{g a s}\right)=0, \quad y=1 .
\end{gathered}
$$

The General solution of the equation (46) is as follows:

$$
\tilde{A}=C_{1} \operatorname{ch} \sqrt{\operatorname{Pr} p} y+C_{2} \operatorname{sh} \sqrt{\operatorname{Pr} p} y+\frac{\sqrt{\operatorname{Pr} p}}{p} \int_{0}^{y} A_{0}(x) \operatorname{sh}[\sqrt{\operatorname{Pr} p}(x-y)] d x
$$

with the constants $C_{1}$ and $C_{2}$, which are defined from boundary conditions (47), (48):

$$
\begin{gathered}
C_{1}=\tilde{A}(p), \\
C_{2}=[\sqrt{\operatorname{Pr} p} \operatorname{ch} \sqrt{\operatorname{Pr} p}+\operatorname{Bish} \sqrt{\operatorname{Pr} p}]^{-1}\left\{\operatorname{Bi} \tilde{A}_{g a s}-\tilde{A}(p)(\sqrt{\operatorname{Pr} p} \operatorname{sh} \sqrt{\operatorname{Pr} p}+\operatorname{Bi} \operatorname{ch} \sqrt{\operatorname{Pr} p})-\right. \\
\left.-\frac{\operatorname{Bi} \sqrt{\operatorname{Pr} p}}{p} \operatorname{Pr} \int_{0}^{1} A_{0}(x) \operatorname{sh}[\sqrt{\operatorname{Pr} p}(x-1)] d x\right\} .
\end{gathered}
$$

The original $A(y, \tau)$ is restored using the formula

$$
A(y, \tau)=\frac{1}{2 \pi i} \int_{l-i \infty}^{l+i \infty} \tilde{A}(y, p) e^{p \tau} d p
$$

The integral (52) is taken along any straight line $\operatorname{Re} p=l>s_{0}$, where $s_{0}$ is the growth index of the function $A(y, \tau)$, and is understood in the sense of the main value.

The task for determining the image $\tilde{B}(y, p)$ is exactly the same as the task (46)-(48) with the replacement of the right part: $-\operatorname{Pr} A_{0}(y)$ for $-\operatorname{Pr} B_{0}(y)-4 \tilde{A}$. Thus, this function is found by the formula:

$$
\begin{aligned}
\tilde{B} & =C_{3} \operatorname{ch} \sqrt{\operatorname{Pr} p} y+C_{4} \operatorname{sh} \sqrt{\operatorname{Pr} p} y+\frac{\sqrt{\operatorname{Pr} p}}{p} \int_{0}^{y} B_{0}(x) \operatorname{sh}[\sqrt{\operatorname{Pr} p}(x-y)] d x- \\
& -\frac{2 C_{1} y}{\sqrt{\operatorname{Pr} p}} \operatorname{ch} \sqrt{\operatorname{Pr} p}-\frac{2 C_{2} y}{\sqrt{\operatorname{Pr} p}} \operatorname{sh} \sqrt{\operatorname{Pr} p}+\frac{2 y}{p} \int_{0}^{y} A_{0}(x) \operatorname{ch}[\sqrt{\operatorname{Pr} p}(x-y)] d x,
\end{aligned}
$$

with constants $C_{3}$ and $C_{4}$ defined from boundary conditions:

$$
\begin{gathered}
C_{3}=\tilde{B}(p), \\
C_{4}=[\sqrt{\operatorname{Pr} p} \operatorname{ch} \sqrt{\operatorname{Pr} p}+\operatorname{Bish} \sqrt{\operatorname{Pr} p}]^{-1}\left\{\operatorname{Bi} \tilde{B}_{\text {gas }}-\tilde{B}(p)(\sqrt{\operatorname{Pr} p} \operatorname{sh} \sqrt{\operatorname{Pr} p}+\operatorname{Bich} \sqrt{\operatorname{Pr} p})+\right. \\
+\operatorname{Pr} \int_{0}^{1} B_{0}(x) \operatorname{ch}[\sqrt{\operatorname{Pr} p}(x-1)] d x-\frac{2(1+\operatorname{Bi})}{p} \int_{0}^{1} A_{0}(x) \operatorname{ch}[\sqrt{\operatorname{Pr} p}(x-1)] d x+ \\
+\frac{2 \sqrt{\operatorname{Pr} p}}{p} \int_{0}^{1} A_{0}(x) \operatorname{sh}[\sqrt{\operatorname{Pr} p}(x-1)] d x+2 C_{1}\left(\frac{\operatorname{ch} \sqrt{\operatorname{Pr} p}}{\sqrt{\operatorname{Pr} p}}+\operatorname{sh} \sqrt{\operatorname{Pr} p}+\frac{\operatorname{Bich} \sqrt{\operatorname{Pr} p}}{\sqrt{\operatorname{Pr} p}}\right)+ \\
\left.+2 C_{2}\left(\frac{\operatorname{sh} \sqrt{\operatorname{Pr} p}}{\sqrt{\operatorname{Pr} p}}+\operatorname{ch} \sqrt{\operatorname{Pr} p}+\frac{\operatorname{Bish} \sqrt{\operatorname{Pr} p}}{\sqrt{\operatorname{Pr} p}}\right)\right\} . \\
-203-
\end{gathered}
$$


You can show using the explicit formulas (49)-(51) and asymptotic representations: $\operatorname{sh} x \sim$ $x+x^{3} / 6, \operatorname{ch} x \sim 1+x^{2} / 2$ for $x \rightarrow 0[20]$, that

$$
\lim _{\tau \rightarrow \infty} A(y, \tau)=\lim _{p \rightarrow 0} p \tilde{A}(y, p)=A^{0}(y),
$$

where $A^{0}(y)$ is a stationary solution for the function $A(y, \tau)$ in (38). When proving, keep in mind that the functions $A_{\text {gas }}(\tau)$ and $A(\tau)$ are originals along with their first derivatives [19] and assume the existence of limits: $\lim _{\tau \rightarrow \infty} A_{\text {gas }}(\tau)=\lim _{p \rightarrow 0} p \tilde{A}_{\text {gas }}(p)=A_{\text {gas }}^{0}, \lim _{\tau \rightarrow \infty} A(\tau)=\lim _{p \rightarrow 0} p \tilde{A}(p)=A$. In addition, the condition (37) must be met.

Similarly, it is shown that

$$
\lim _{\tau \rightarrow \infty} B(y, \tau)=\lim _{p \rightarrow 0} p \tilde{B}(y, p)=B^{0}(y),
$$

that is, as time increases, the temperature perturbation becomes stationary, provided that the functions $B_{g a s}(\tau)$ and $B(\tau)$ are originals along with their first derivatives and there are limits: $\lim _{\tau \rightarrow \infty} B_{\text {gas }}(\tau)=\lim _{p \rightarrow 0} p \tilde{B}_{\text {gas }}(p)=B_{\text {gas }}^{0}, \lim _{\tau \rightarrow \infty} B(\tau)=\lim _{p \rightarrow 0} p \tilde{B}(p)=B$.

Thus, the fair

Theorem 1. Problem solving for the functions $A(y, \tau), B(y, \tau)$ are determined by the inverse Laplace transform by the formulas (49), (53), and with the growth of time, they reach a stationary regime, if $A_{\text {gas }}(\tau) \rightarrow A_{\text {gas }}^{0}, B_{\text {gas }}(\tau) \rightarrow B_{\text {gas }}^{0}, A(\tau) \rightarrow A, B(\tau) \rightarrow B$ when $\tau \rightarrow \infty$ and the condition (37) is met.

\section{Determination of the mixture concentration}

Applying to the initial-boundary problem for the concentration the mixture of Laplace transform, obtain for the image $\tilde{H}(y, p)$ task

$$
\begin{gathered}
\tilde{H}_{y y}-\operatorname{Scp} \tilde{H}=-\operatorname{Sc} H_{0}(y)+\operatorname{Sr} \operatorname{Pr} A_{0}(y)-\operatorname{SrPr} p \tilde{A}, \quad 0<y<1 ; \\
\tilde{H}_{y}+\operatorname{Sr} \tilde{A}_{y}=0, \quad y=0 ; \\
\tilde{H}_{y}+\operatorname{Sr} \tilde{A}_{y}=0, \quad y=1 .
\end{gathered}
$$

The General solution of the equation (56) for $\operatorname{Pr} \neq \mathrm{Sc}$ is as follows:

$$
\begin{gathered}
\tilde{H}=C_{5} \operatorname{ch} \sqrt{\operatorname{Sc} p} y+C_{6} \operatorname{sh} \sqrt{\operatorname{Sc} p} y+ \\
+\frac{1}{\sqrt{\operatorname{Sc} p}} \int_{0}^{y}\left(\operatorname{Sc} H_{0}(x)-\operatorname{SrPr} A_{0}(x)\right) \operatorname{sh}[\sqrt{\operatorname{Sc} p}(x-y)] d x- \\
-\frac{\operatorname{SrPr}}{\operatorname{Pr}-\operatorname{Sc}}\left(C_{1} \operatorname{ch} \sqrt{\operatorname{Pr} p} y+C_{2} \operatorname{sh} \sqrt{\operatorname{Pr} p} y+\frac{\sqrt{\operatorname{Pr} p}}{p} \int_{0}^{y} A_{0}(x) \operatorname{sh}[\sqrt{\operatorname{Pr} p}(x-y)] d x\right),
\end{gathered}
$$

with constants $C_{5}$ and $C_{6}$ defined from boundary conditions (57), (58):

$$
C_{6}=\frac{C_{2} \operatorname{Sr} \sqrt{\operatorname{PrSc}}}{\operatorname{Pr}-\mathrm{Sc}}
$$




$$
\begin{aligned}
C_{5}= & {[\sqrt{\operatorname{Sc} p} \operatorname{sh} \sqrt{\operatorname{Sc} p}]^{-1}\left\{\int_{0}^{1}\left(\operatorname{Sc} H_{0}(x)-\operatorname{SrPr} A_{0}(x)\right) \operatorname{ch}[\sqrt{\operatorname{Sc} p}(x-1)] d x+\right.} \\
& +\frac{\operatorname{SrSc}}{\operatorname{Pr}-\operatorname{Sc}}\left[\sqrt{\operatorname{Pr} p}\left(C_{1} \operatorname{sh} \sqrt{\operatorname{Pr} p}+C_{2} \operatorname{ch} \sqrt{\operatorname{Pr} p}\right)-\right. \\
& \left.-\operatorname{Pr} \int_{0}^{1} A_{0}(x) \operatorname{ch}[\sqrt{\operatorname{Pr} p}(x-1)] d x\right\}-\frac{C_{2} \operatorname{Sr} \sqrt{\operatorname{PrSc}}}{\operatorname{Pr}-\operatorname{Sc}} \operatorname{cth} \sqrt{\operatorname{Sc} p}
\end{aligned}
$$

The task for defining an image $\tilde{G}(y, p)$ is exactly the same as the task (56)-(58) with replacing the right part: $-\operatorname{Sc} H_{0}(y)+\operatorname{SrPr} A_{0}(y)-\operatorname{SrPr} p \tilde{A}$ for $-\operatorname{Sc} G_{0}(y)+\operatorname{SrPr} B_{0}(y)-\operatorname{SrPr} p \tilde{B}-4 \tilde{H}$.

The General solution for $\tilde{G}(y, p)$ when $\operatorname{Pr} \neq \mathrm{Sc}$ has the form:

$$
\begin{gathered}
\tilde{G}=C_{7} \operatorname{ch} \sqrt{\operatorname{Sc} p} y+C_{8} \operatorname{sh} \sqrt{\operatorname{Sc} p} y+ \\
+\frac{1}{\sqrt{\operatorname{Sc} p}} \int_{0}^{y}\left(\operatorname{Sc} G_{0}(x)-\operatorname{SrPr} B_{0}(x)\right) \operatorname{sh}[\sqrt{\operatorname{Sc} p}(x-y)] d x- \\
-\frac{2 C_{5} y}{\sqrt{\operatorname{Sc} p}} \operatorname{ch} \sqrt{\operatorname{Sc} p} y-\frac{2 C_{6} y}{\sqrt{\operatorname{Sc} p}} \operatorname{sh} \sqrt{\operatorname{Sc} p} y- \\
-\frac{\operatorname{SrPr}}{\operatorname{Pr}-\operatorname{Sc}}\left(C_{3} \operatorname{ch} \sqrt{\operatorname{Pr} p} y+C_{4} \operatorname{sh} \sqrt{\operatorname{Pr} p} y+\frac{\sqrt{\operatorname{Pr} p}}{p} \int_{0}^{y} B_{0}(x) \operatorname{sh}[\sqrt{\operatorname{Pr} p}(x-y)] d x-\right. \\
\left.-\frac{2 C_{1} y}{\sqrt{\operatorname{Pr} p}} \operatorname{ch} \sqrt{\operatorname{Pr} p} y-\frac{2 C_{2} y}{\sqrt{\operatorname{Pr} p}} \operatorname{sh} \sqrt{\operatorname{Pr} p} y+\frac{2 y}{p} \int_{0}^{y} A_{0}(x) \operatorname{ch}[\sqrt{\operatorname{Pr} p}(x-y)] d x\right)+ \\
+\frac{2 y}{\operatorname{Sc} p} \int_{0}^{y}\left(\operatorname{Sc} H_{0}(x)-\operatorname{SrPr} A_{0}(x)\right) \operatorname{ch}[\sqrt{\operatorname{Sc} p}(x-y)] d x
\end{gathered}
$$

where the constants $C_{7}$ and $C_{8}$ are defined from the boundary conditions as follows:

$$
\begin{aligned}
& C_{8}=\frac{2 C_{5}}{\operatorname{Sc} p}+\frac{\operatorname{SrSc}\left(\operatorname{Pr} p C_{4}-2 C_{1}\right)}{\sqrt{\operatorname{PrSc} p}(\operatorname{Pr}-\mathrm{Sc})}, \\
& C_{7}=[\sqrt{\operatorname{Sc} p} \operatorname{sh} \sqrt{\operatorname{Sc} p}]^{-1}\left\{2 C_{5}\left(\frac{\operatorname{ch} \sqrt{\operatorname{Sc} p}}{\sqrt{\operatorname{Sc} p}}+\operatorname{sh} \sqrt{\operatorname{Sc} p}\right)+2 C_{6}\left(\frac{\operatorname{sh} \sqrt{\operatorname{Sc} p}}{\sqrt{\operatorname{Sc} p}}+\operatorname{ch} \sqrt{\operatorname{Sc} p}\right)+\right. \\
& +\int_{0}^{1}\left(\operatorname{Sc} G_{0}(x)-\operatorname{SrPr} B_{0}(x)\right) \operatorname{ch}[\sqrt{\operatorname{Sc} p}(x-1)] d x+ \\
& +\frac{\mathrm{SrSc}}{\operatorname{Pr}-\mathrm{Sc}}\left[\sqrt{\operatorname{Pr} p}\left(C_{3} \operatorname{sh} \sqrt{\operatorname{Pr} p}+C_{4} \operatorname{ch} \sqrt{\operatorname{Pr} p}\right)-\operatorname{Pr} \int_{0}^{1} B_{0}(x) \operatorname{ch}[\sqrt{\operatorname{Pr} p}(x-1)] d x-\right. \\
& -2 C_{1}\left(\frac{\operatorname{ch} \sqrt{\operatorname{Pr} p}}{\sqrt{\operatorname{Pr} p}}+\operatorname{sh} \sqrt{\operatorname{Pr} p}\right)-2 C_{2}\left(\frac{\operatorname{sh} \sqrt{\operatorname{Pr} p}}{\sqrt{\operatorname{Sc} p}}+\operatorname{ch} \sqrt{\operatorname{Pr} p}\right)+ \\
& \left.+\frac{2}{p} \int_{0}^{1} A_{0}(x) \operatorname{ch}[\sqrt{\operatorname{Pr} p}(x-1)] d x-\frac{2 \sqrt{\operatorname{Pr} p}}{p} \int_{0}^{1} A_{0}(x) \operatorname{sh}[\sqrt{\operatorname{Pr} p}(x-1)] d x\right]- \\
& -\frac{2}{\operatorname{Sc} p} \int_{0}^{1}\left(\operatorname{Sc} H_{0}(x)-\operatorname{SrPr} A_{0}(x)\right) \operatorname{ch}[\sqrt{\operatorname{Sc} p}(x-1)] d x+ \\
& \left.+\frac{2}{\sqrt{\operatorname{Sc} p}} \int_{0}^{1}\left(\operatorname{Sc} H_{0}(x)-\operatorname{SrPr} A_{0}(x)\right) \operatorname{sh}[\sqrt{\operatorname{Sc} p}(x-1)] d x\right\}-C_{8} \operatorname{cth} \sqrt{\operatorname{Sc} p} .
\end{aligned}
$$


You can show using the formulas (59)-(61) that

$$
\lim _{\tau \rightarrow \infty} H(y, \tau)=\lim _{p \rightarrow 0} p \tilde{H}(y, p)=H^{0}(y)
$$

where $H^{0}(y)$ is a stationary solution for the function $H(y, \tau)$ in $(39)$. When output, you must again assume that there are limits: $\lim _{\tau \rightarrow \infty} A_{\text {gas }}(\tau)=A_{\text {gas }}^{0}, \lim _{\tau \rightarrow \infty} A(\tau)=A$. In addition, the condition (37) must be met.

Similarly, it is shown that

$$
\lim _{\tau \rightarrow \infty} G(y, \tau)=\lim _{p \rightarrow 0} p \tilde{G}(y, p)=G^{0}(y),
$$

where $G^{0}(y)$ is a stationary distribution for the function $G(y, \tau)$.

Thus, the fair

Theorem 2. Problem solving for the functions $H(y, \tau), G(y, \tau)$ are determined by the inverse Laplace transform by the formulas (59), (62), and with the growth of time, they reach a stationary regime, if $A_{\text {gas }}(\tau) \rightarrow A_{\text {gas }}^{0}, B_{\text {gas }}(\tau) \rightarrow B_{\text {gas }}^{0}, A(\tau) \rightarrow A, B(\tau) \rightarrow B$ when $\tau \rightarrow \infty$ and the condition (37) is met.

\section{Determination of the velocity field}

Applying the Laplace transform to a problem for speed reduces it to a boundary value problem for an ordinary differential equation

$$
\begin{gathered}
\tilde{U}_{y y}-p \tilde{U}=-U_{0}(r)-\tilde{F}(p), \quad 0<y<1 ; \\
\tilde{U}(0, p)=0, \quad y=0 \\
\int_{0}^{1} \tilde{U} d y=0, \quad y=1 ; \\
\tilde{U}_{y}=-2(\tilde{A}+\mathrm{M} \tilde{H}), \quad y=1 .
\end{gathered}
$$

The General solution of the equation (65) is written as follows:

$$
\tilde{U}=C_{9} \operatorname{ch} \sqrt{p} y+C_{10} \operatorname{sh} \sqrt{p} y+\frac{1}{\sqrt{p}} \int_{0}^{y} U_{0}(x) \operatorname{sh}[\sqrt{p}(x-y)] d x,
$$

with constants $C_{9}$ and $C_{10}$ defined from boundary conditions (66)-(68):

$$
\begin{gathered}
C_{9}=-\frac{\tilde{F}(p)}{p} \\
C_{10}=\frac{2(\tilde{A}+\mathrm{M} \tilde{H})+\sqrt{p} \int_{0}^{1} U_{0}(x) \operatorname{ch} \sqrt{p}(x-1) d x+\tilde{F}(p) \operatorname{sh} \sqrt{p}}{p \operatorname{ch} \sqrt{p}}
\end{gathered}
$$

where the functions $\tilde{A}(y, p), \tilde{H}(y, p)$ are given by the formulas (49), (59) for $y=1$, and the pressure gradient $\tilde{F}(p)$ is as follows:

$$
\begin{gathered}
\tilde{F}(p)=[\sqrt{p} \operatorname{ch} \sqrt{p}-\operatorname{sh} \sqrt{p}]^{-1}\{\sqrt{p}(\operatorname{ch} \sqrt{p}-1)(2 \tilde{A}+2 \mathrm{M} \tilde{H}- \\
\left.\left.-\int_{0}^{1} U_{0}(x) \operatorname{ch} \sqrt{p}(x-1) d x\right)-p \operatorname{ch} \sqrt{p} \int_{0}^{1}\left[\int_{0}^{y} U_{0}(x) \operatorname{ch} \sqrt{p}(x-1) d x\right] d y\right\} .
\end{gathered}
$$


You can derive equality from the expressions (69)-(72):

$$
\lim _{p \rightarrow 0} p \widetilde{U}(r, p)=U^{0}(y)
$$

where $U^{0}(y)$ is a stationary velocity distribution from (40). When you output (73), you must assume the existence of the limits: $\lim _{\tau \rightarrow \infty} A_{\text {gas }}(\tau)=A_{\text {gas }}^{0}, \lim _{\tau \rightarrow \infty} B_{\text {gas }}(\tau)=B_{\text {gas }}^{0}, \lim _{\tau \rightarrow \infty} A(\tau)=A$, $\lim _{\tau \rightarrow \infty} B(\tau)=B$ and the fulfillment of the condition (37).

$\rightarrow \infty$ Thus, the fair

Theorem 3. Problem solving for the function $U(y, \tau)$ is determined by the inverse Laplace transform by the formulas (69), (71), and with the growth of time, they reach a stationary regime, if $A_{\text {gas }}(\tau) \rightarrow A_{\text {gas }}^{0}, B_{\text {gas }}(\tau) \rightarrow B_{\text {gas }}^{0}, A(\tau) \rightarrow A, B(\tau) \rightarrow B$ when $\tau \rightarrow \infty$ and the condition (37) is met.

\section{Numerical solution}

The obtained formulas in the Laplace images were used to find the temperature, concentration, and velocity fields of the mixture under certain conditions imposed on the external temperature $A_{\text {gas }}(\tau)$ and the solid wall temperature $A(\tau)$. In this purpose, the numerical method of the inverse Laplace transform was used using the quadrature formula of the highest degree of accuracy, constructed for the Riemann-Mellin integral [21]:

$$
f(t)=\frac{1}{2 \pi i} \int_{c-i \infty}^{c+i \infty} F(\sigma) e^{\sigma t} d \sigma .
$$

Let the image function $F(\sigma)$ is regular in the half-plane $\operatorname{Re} \sigma>\alpha$. Replacing $\sigma=p / t+\alpha$ converts (74) to an integral

$$
f(t)=\frac{1}{2 \pi i} \frac{e^{\alpha t}}{t} \int_{\varepsilon-i \infty}^{\varepsilon+i \infty} F^{*}(p) e^{p} d p,
$$

here $\varepsilon$ - any small positive number, and $F^{*}(p)=F(p / t+\alpha)=F(\sigma)$. It is assumed that $F^{*}(p)$ has the form $F^{*}(p)=\varphi(p) / p^{k}$, here $k>0, \varphi(p)$ is regular in the half-plane Re $p>0$ and there is $\lim _{t \rightarrow \infty} \varphi(p) \neq 0 ; \infty$. Then the quadrature formula of the highest degree of accuracy is applied to the integral

$$
\frac{1}{2 \pi i} \int_{\varepsilon-i \infty}^{\varepsilon+i \infty} \varphi(p) \frac{e^{p}}{p^{k}} d p
$$

which has the form

$$
\frac{1}{2 \pi i} \int_{\varepsilon-i \infty}^{\varepsilon+i \infty} \varphi(p) \frac{e^{p}}{p^{k}} d p \simeq \sum_{m=1}^{n} A_{m} \varphi\left(p_{m}\right),
$$

and since

$$
\varphi\left(p_{m}\right)=p_{m}^{k} F^{*}\left(p_{m}\right)=p_{m}^{k} F\left(p_{m} / t+\alpha\right),
$$

then

$$
f(t) \simeq \frac{e^{\alpha t}}{t} \sum_{m=1}^{n} A_{m} p_{m}^{k} F\left(p_{m} / t+\alpha\right),
$$


moreover, the coefficients $A_{m}$ and $p_{m}$ nodes depend on $k$ и $n$. The formula (77) was the basis of a program that performs the inverse Laplace transform. The coefficients $A_{m}$ and the nodes $p_{m}$ were taken from [22].

Using the numerical method, quantitative results were obtained for a model system with the following parameter values: $A_{\text {gas }}^{0}=0.2, A=-0.1, \mathrm{Sr}=3, \mathrm{Bi}=2, \mathrm{Pr}=2, \mathrm{Sc}=1, \mathrm{M}=100$, $A_{\text {gas }}(\tau)=A_{\text {gas }}^{0}+\exp (-\lambda \tau) \sin (\omega \tau), A(\tau)=A+\exp (-\lambda \tau) \sin (\omega \tau)$, here $\omega=1$. Fig. 2-7 shows the evolution of dimensionless profiles of temperature, concentration, and velocity of the mixture at different times.
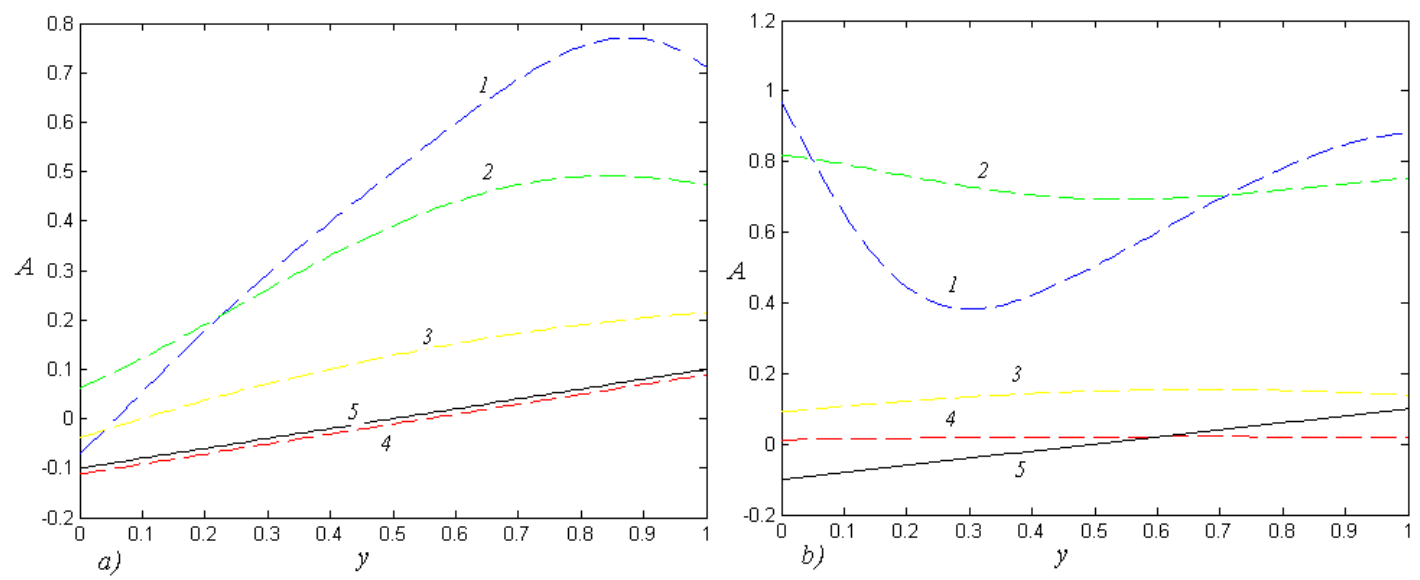

Fig. 2. The temperature profile at $\lambda=1: 1-\tau=0.02,2-\tau=0.2,3-\tau=2.4,4-\tau=4.5$, 5 - the stationary solution
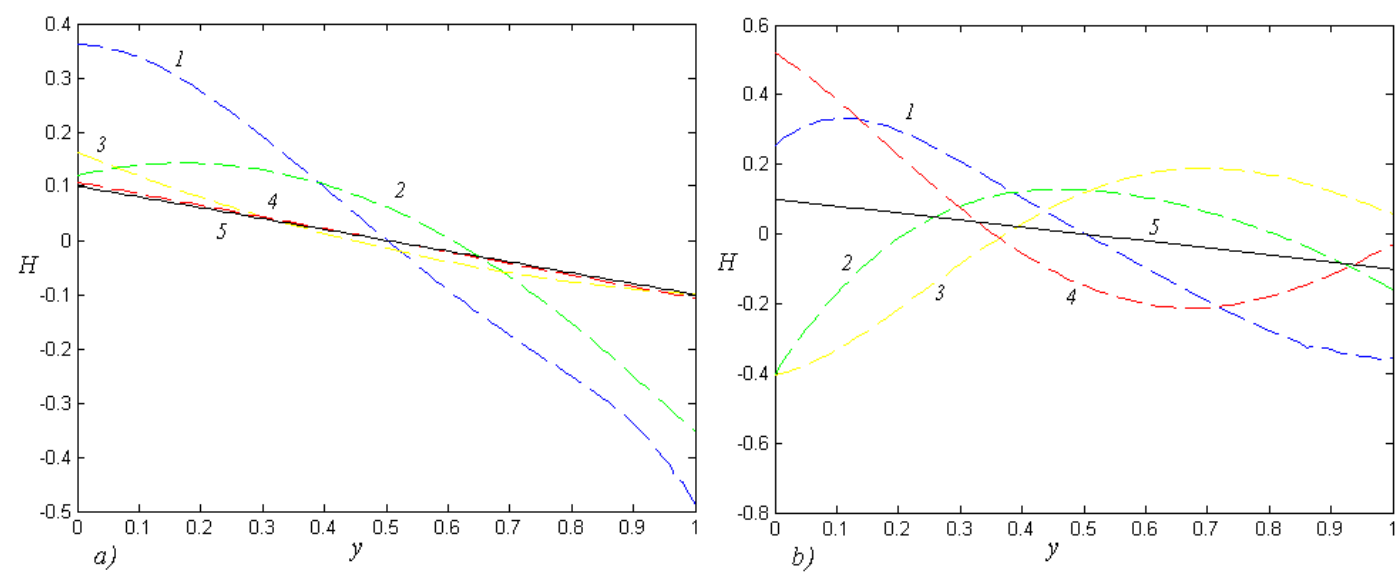

Fig. 3. The concentration profile at $\lambda=1: 1-\tau=0.02,2-\tau=0.2,3-\tau=1.7,4-\tau=4.8$, 5 - the stationary solution

If the functions $A_{\text {gas }}(\tau), A(\tau)$ have finite limits at $\tau \rightarrow \infty$, equal to $A_{\text {gas }}^{0}$ and $A$, respectively, and the condition (37) is met, then there is convergence to the stationary distribution (see Fig. $2 a, 3 a, 4 a$ at $\lambda=1$ ). If these functions have no limits at $\tau \rightarrow \infty$ (either the limits exist, but the connection between $A_{\text {gas }}^{0}$ and $\mathrm{A}$ is broken), then non-stationary solutions do not converge to stationary solutions with increasing time (see Fig. 2b, 3b, 4b at $\lambda=1$ ). 

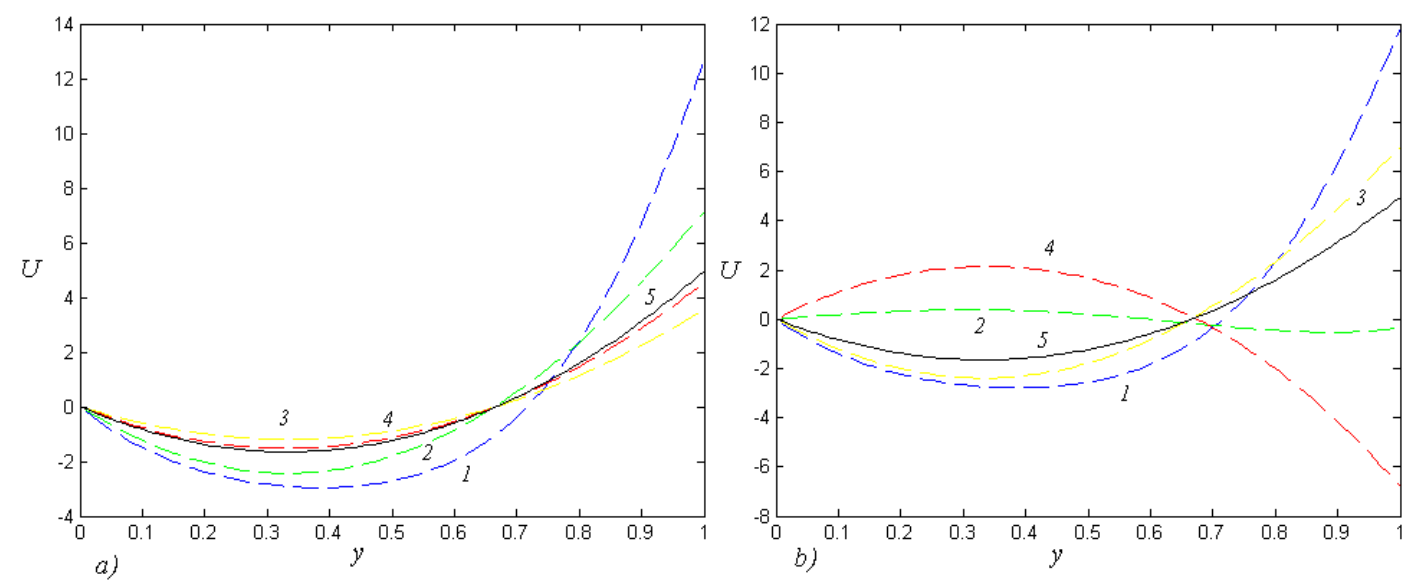

Fig. 4. The velocity profile at $\lambda=1: 1-\tau=0.04,2-\tau=1.0,3-\tau=1.4, \quad 4-\tau=3.14$, 5 - the stationary solution
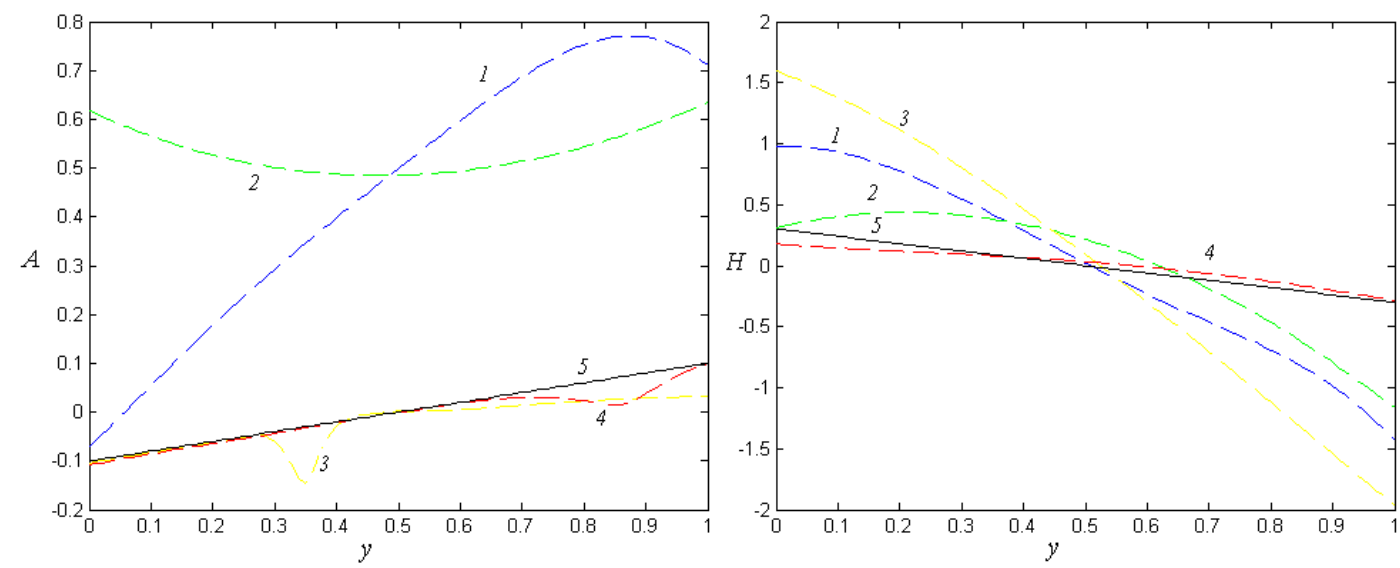

Fig. 5. The temperature and concentration profiles at $\lambda=10^{-3}: 1-\tau=0.03,2-\tau=0.3$, $3-\tau=35.6,4-\tau=37.85,5-$ the stationary solution

For Fig. 5, 6 presents temperature, concentration, and velocity profiles for $\lambda=10^{-3}$. It takes a longer period of time for the solution to return to the steady state, and there are fluctuations. The dependence of the speed $U(y, \tau)$ on the parameter $\mathrm{M}$ was also studied (see Fig. 7). It turned out that the non-stationary solution quickly switches to the stationary regime for any $\mathrm{M}$.

Analyzing the numerical solution for the function $U(y, \tau)$, we conclude that she takes a minimum value for $y=1 / 3$, as well as $U<0$ for $0<y<2 / 3$ and $U>0$ for $2 / 3<y<1$, which corresponds to the result obtained in the formula (40). It follows that the current changes direction at a depth equal to $2 / 3$ of the thickness of the liquid layer.

Fig. 8 shows the trajectories of liquid particles (current lines) and the surface of the current when moving a viscous heat-conducting binary mixture with a flat free boundary. It can be seen that there is a return rotationally-symmetric flow of the liquid, which occurs under the influence of a pressure gradient. The resulting motion is a vortex in the $r y$ plane with the center shifted to the free boundary. In this case, the maximum speed is achieved on a free surface.

Let's see what happens to the rest of the required functions. As a result of heat exposure, 


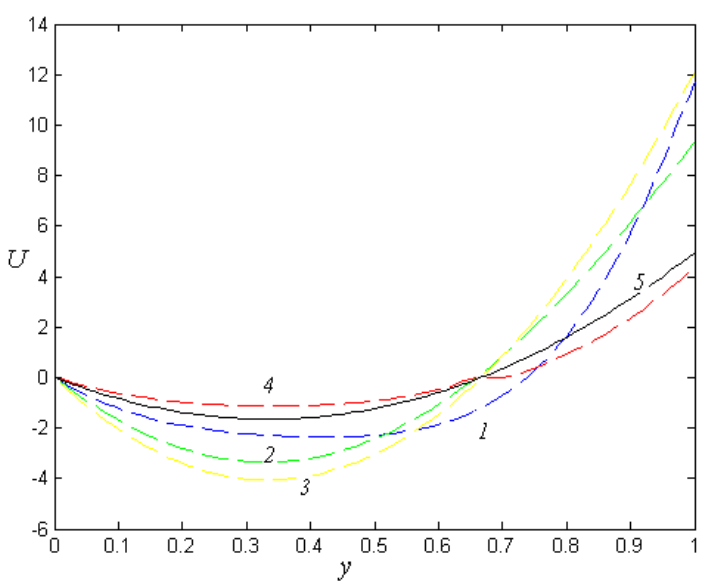

Fig. 6. The velocity profile at $\lambda=10^{-3}: 1-\tau=0.5,2-\tau=4.5,3-\tau=35.2,4-\tau=37.8$, 5 - the stationary solution
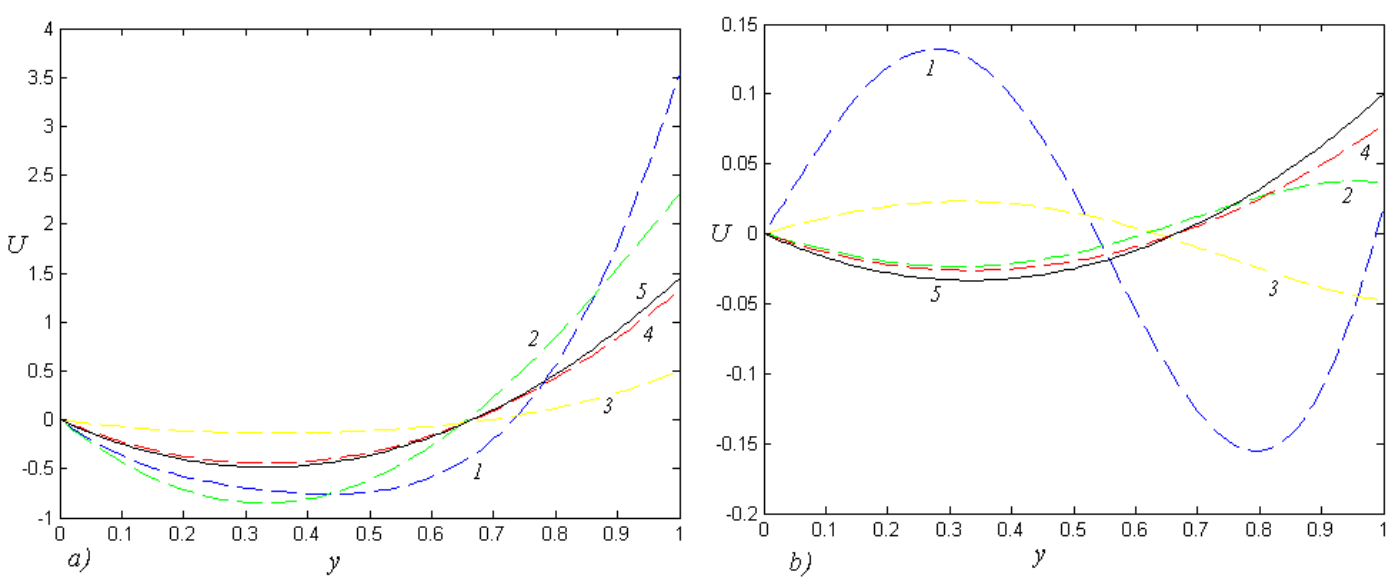

Fig. 7. The velocity profile for different values of the parameter $\mathrm{M}: a) \mathrm{M}=10, b) \mathrm{M}=1$
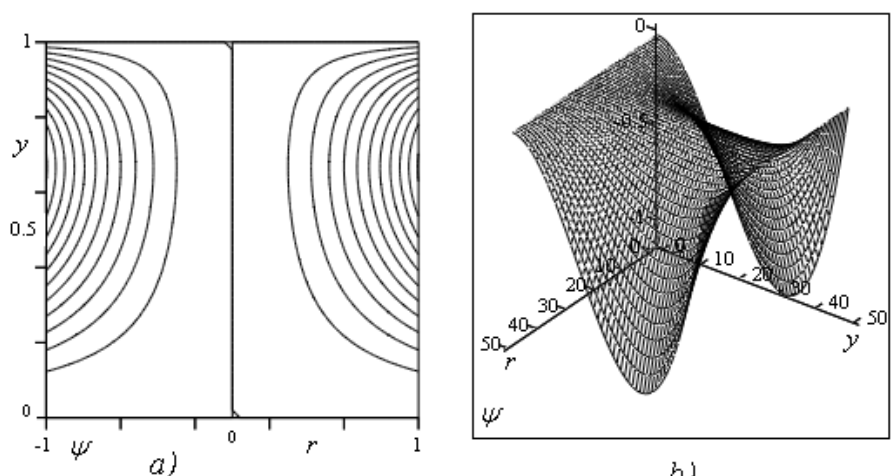

b)

Fig. 8. a) the trajectories of liquid particles, $b$ ) the surface current 
the temperature $A(y, \tau)$ increases and the concentration $H(y, \tau)$ decreases. There is a thermodiffusion effect-the Soret effect. Anomalous thermodiffusion occurs, in which light components tend to move to colder areas, and heavy components end up in areas with increased temperature (since $c$ in the system (1) is the concentration of the light component).

\section{Conclusion}

Rotationally-symmetric motion of a binary mixture with a flat free boundary at small Marangoni numbers is investigated. The problem is reduced to the inverse linear initial-boundary value problem for parabolic equations. Using Laplace transformation properties the exact analytical solution is obtained. It is shown that a stationary solution is the limiting one with the growth of time if there is a certain relationship between the temperature of the solid wall and the external temperature of the gas. If there is no connection, the convergence to the stationary solution is broken. Some examples of numerical reconstruction of the temperature, concentration and velocity fields are given, which confirm the theoretical conclusions.

\section{References}

[1] N.Darabi, Journal of the Siberian Federal University. Mathematics and physics, 8(2015), no. $3,260-272$.

[2] R.V.Birikh, J. Appl. Mech. Tech. Phys., 7(1966), 43-44. DOI: 10.1007/BF00914697

[3] A.G.Kirdyashkin, Thermogravitational and thermocapillary convection in a horizontal fluid layer, In: Fluid mechanics and transport processes in zero gravity, Sverdlovsk, UNC of the USSR Academy of Sciences, 1983, 81-86 (in Russian).

[4] A.G.Kirdyashkin, International Journal of Heat and Mass Transfer, 27(1984), no. 8, 1205-1218. DOI: 10.1016/0017-9310(84)90048-6

[5] A.G.Kirdyashkin, Thermocapillary periodic flows, Preprint no. 8. Novosibirsk: IGiG SO AS USSR, 1985 (in Russian).

[6] A.F.Sidorov, J. Appl. Mech. Tech. Phys., 30(1989), 197-203. DOI: 10.1007/BF00852164

[7] S.N.Aristov, K.G.Shvartz, Vortex flows of advective nature in a rotating liquid layer, Perm, PSU publishing House, 2006 (in Russian).

[8] O.N.Goncharova, O.A.Kabov, Microgravity Sci. Thechnol., 21(2009), 129-137. DOI: $10.1007 / \mathrm{s} 12217-009-9108-\mathrm{x}$

[9] V.K.Andreev, Birikh's Solution of convection equations and some of its generalizations, Preprint no. 1-10, Krasnoyarsk, Institute of Computational Modeling SB RAS, 2010 (in Russian).

[10] S.N.Aristov, K.G.Schwartz, Vortex flows in thin layers of liquid, Kirov, VyatSU, 2011 (in Russian).

[11] V.V.Pukhnachev, The Bulletin of Irkutsk State University. Series Mathematics, 3(2011), no. 1, 61-69 (in Russian).

[12] V.K.Andreev, V.B.Bekezhanova, J. Appl. Mech. Tech. Phy., 54(2013), 171-184. DOI: $10.1134 / \mathrm{S} 0021894413020016$ 
[13] S.N.Aristov, E.Yu.Prosviryakov, J. Samara State Tech. Univ., Ser. Phys. Math. Sci., 32(2013), no. 3, 110-118 (in Russian). DOI: 10.14498/vsgtu1205

[14] A.V.Gorshkov, E.Yu.Prosviryakov, Computer research and modeling, 8(2016), no. 6, 927940 (in Russian). DOI: 10.20537/2076-7633-2016-8-6-927-940

[15] V.V.Privalova, E.Yu.Prosviryakov, J. Samara State Tech. Univ., Ser. Phys. Math. Sci., 22(2018), no. 3, 532-548. DOI: 10.14498/vsgtu1638

[16] K.Hiemenz, Die Grenzschicht an einem in den gleichformigen Flussigkeitsstrom eingetauchten geraden Kreiszylinder, Dinglers Politech. J., 326(1911), 321.

[17] V.K.Andreev, V.E.Zachvataev, E.A.Ryabitskiy, Thermocapillary instability, Novosibirsk, Nauka, 2000 (in Russian).

[18] L.V.Ovsyannikov, Group analysis of differential equations, Moscow, Nauka, 1978 (in Russian).

[19] M.A.Lavrentiev, B.V.Shabat, Methods of the theory of functions of a complex variable, Moscow, Nauka, 1973 (in Russian).

[20] M.Abramovits, I.Stigan, Handbook of special functions, Moscow, Nauka, 1979 (in Russian).

[21] V.I.Krylov, N.S.Skoblya, Reference book on the numerical inversion of the Laplace transform, Minsk, Science and technology, 1968 (in Russian).

[22] A.P.Prudnikov, V.A.Ditkin, Operational calculus, Moscow, Higher school, 1975 (in Russian).

\title{
Вращательно-осесимметричное движение бинарной смеси с плоской свободной границей при малых числах Марангони
}

\author{
Виктор К. Андреев \\ Институт вычислительного моделирования СО РАН \\ Красноярск, Российская Федерация \\ Сибирский федеральный университет \\ Российская Федерация \\ Наталья Л. Собачкина \\ Сибирский федеральный университет \\ Российская Федерация
}

\begin{abstract}
Аннотация. Исследовано вращательно-симметричное движение плоского слоя бинарной смеси со свободной границей при малых числах Марангони. Задача сводится к обратной линейной начальнокраевой задаче для параболических уравнений. В изображениях по Лапласу получено точное аналитическое решение. Найдено стационарное решение задачи и доказано, что оно является предельным с ростом времени при условии существования определенной связи между температурой твердой стенки и внешней температурой газа. В случае отсутствия связи сходимость к стационарному решению нарушается. Приведены примеры численного восстановления полей температуры, концентрации и скорости, подтверждающие теоретические выводы.
\end{abstract}

Ключевые слова: бинарная смесь, свободная граница, обратная задача, градиент давления, стационарное решение, преобразование Лапласа, тепловое число Марангони. 